\title{
Book Review: Maurizio Gotti and Carmen Sancho Guinda (eds). (2013). Narratives in Academic and Professional Genres. Bern: Peter Lang, pp 511.
}

\author{
Yolanda Morató ${ }^{1}$ \\ Universidad de León
}

Whereas academic discourse has been extensively researched in the last three decades, a neighbouring area, namely professional genres, has not received due attention, at least not to the same degree. In 1990 John Swales' Genre Analyses: English in Academic and Research Settings set the pace for the formal analysis of these specific research contexts and focused on contemporary genres in the academic world. Its functional components or "moves" were described as the essential elements that any genre should comprise. Now, more than twenty years after Swales' survey of approaches to varieties of language and genres, a new volume on the subject, edited by Carmen Sancho Guinda and Maurizio Gotti, not only reassesses the role of narratives within academic genres but also offers a fresh approach to professional practice taking into account multidisciplinary corpora, different settings and a thorough rereading of some key concepts for their analysis.

As genre repertoires tend to overlap more and more (Orlikowski and Yates, 1994), the concept of genre, with its various definitions and major effects, is worth a comprehensive analysis. Swales (1990) defined it as "a class of communicative events, the members of which share some set of communicative purposes" (p. 58). However, for Sancho Guinda and Gotti, Swales' "labelling of narrative as a pre-genre" poses several questions regarding genre families, text typologies and, ultimately, the overlapping spaces of narratives, which for them result into "the hybridisation and bending

Corresponding author - University of León. Email: ymora@unileon.es 
of genres" (p. 17). Their aim is to expand the concept of narrative in order to "call for insertion within a larger conceptual network to help develop a more fluid model" (p. 14). The idea is to further explore the narrative phenomenon, that is, to broaden the analyses previously offered by Swales (1990), Martin and Rose (2003, 2008), and Biber and Conrad (2009). To start with, it is necessary to note that the term 'narrative(s)' is a slippery concept which many have endeavoured to define from the point of view of literary discourse and, more recently, from other disciplines such as marketing and advertising, which are increasingly using linguistic strategies and literary techniques with the purpose of getting closer to their target audiences. In addition, spoken corpora demonstrate that oral discourse in academic and professional settings complies with, and in some cases exceeds, those "linguistic markers of narrativity" which have been used to classify a set of written texts in the past. With such possibilities at hand, Narratives in Academic and Professional Genres displays a whole range of oral and textual dynamics through the contributions, which are divided into two distinct parts: 'Narratives in Academic Genres', consisting of eleven chapters, and 'Narratives in Professional Genres', with nine chapters. This two-sided approach allows the editors of this volume to cover different types of academic and professional narratives from a broad range of perspectives and applications.

In an introductory chapter titled 'Weaving a Narrative Paradigm in Academic and Professional Communication', which comes right after the brief but encouraging foreword contributed by Vijay Bhatia, the editors present a range of approaches, contextualising them within the discipline of Narratology; the use of its "tools and foci" is aimed at "synergising theoretical positions and opening research windows that otherwise would be less prominent in specialised communication" (p. 13). Similarly, Brian Paltridge adds the final touch to this selection by challenging stock interpretations of academic and professional genres in his afterword. Focusing on the importance of narrative inquiry and its ability to "provide insights into people's beliefs and experiences" (p. 497), Paltridge looks at those events and experiences that people leave out when reconstructing and interpreting who they are or what they write about. For this purpose, he quotes useful representative contributions from a good number of scholars, from early-stage to senior positions.

Over half of this comprehensive book is devoted to locate and analyse "discursive practices of a number of disciplinary cultures" (p. 25). To a large extent, the first section can be divided into those chapters with a focus on oral channels (e.g. conference presentations, meeting minutes, and academic 
discourses), on written discourse (e.g. research articles, blogs, and book reviews), and, finally, on visual media (e.g. video essays and digital video documentaries). An interesting aspect here is how apparently different media converge in their everyday practice. For instance, as Anna Mauranen argues in her chapter, "'But then when I started to think...: Narrative Elements in Conference Presentations", there is a close proximity between research blogs and the structure of a conference presentation. Conventions of written academic prose are present in research blogs both at their presentation stage and at their discussion level, in which register is lowered and formal structures are replaced by question and answer dynamics. This is a highly interesting point considering that the following chapter in this section, "Narratives in Academic Blogs", by María José Luzón, explicitly focuses on this medium. Rosa Lorés also delves into the overlapping nature of narration and evaluation through her selection of book reviews in "The Same Story? Enhancing Membership and Constructing Knowledge in Spanish and English History Book Reviews".

The use of pronouns as agents and subjects of narratives is broadly covered in several articles; an illustration of this is Mauranen's approach, which is complemented by other essays such as Carmen Daniela Maier and Jan Engberg's "Tendencies in the Multimodal Evolution of Narrator's Types and Roles in Research Genres". Other linguistic elements are examined in Marina Bondi's "Historians as Recounters: Description across Genres", in which she explores a corpus of papers to study deixis, applied to people, places and time. Description plays an essential role here, since it contributes to understand the reader's mental reconstruction and, subsequently, his or her explicit construction of perspective and position. Similarly, though placed as the last chapter of the second section, Ismael Arinas Pellón's "How do you Read a U.S. Patent? Motivation for Descriptions of Intellectual Property and its 'Metes and Bounds'" examines the role of pronouns in patents. Arinas, following Bazerman (1999), describes these documents as "part of a process institutionalised to obtain property", with a particular reader in mind, therefore, manifesting a certain degree of vagueness. "A Tale of Two Genres: Narrative Structure in Students' Scientific Writing", by Christoph A. Hafner, Lindsay Miller and Connie Ng Kwai-Fun, also considers key questions related to the reception of texts, for instance, topic sophistication, different audiences, and competing narratives within the "narrative of science". They observe that there are "discursive differences in scientific writing for specialist and nonspecialist audiences" (p. 237), which inevitably have an impact on the acquisition of "discipline-specific literacy in a given scientific field" (p. 253). 
Two chapters appropriately tackle two academic physical settings in which specific types of narratives take place. Begoña Bellés deals with "Marginal Stories in Classroom Asides" while Christine Feak offers "Insights into the Academy: Narratives in and of Public Meetings of the University". Bellés presents highly interesting conclusions, as she notices how lecture asides tend to embody an evaluative aspect, especially when student interaction is involved; besides, they are more abundant during the first 20-30 minutes. Feak uses the official minutes from four open public meetings in a major US university, together with her observations, to conclude that these meetings share a ritual character, so it is difficult to state their "transparency in university decision-making and operations" (p. 99). Other institutional insights include Luisa Caiazzo's "Factual Reporting in the 'About' Page of British University Websites" and a publication-oriented examination: Pilar Mur Dueñas's article, "Scholars Recounting their Own Research in Journal Articles: An Intercultural (English-Spanish) Perspective". Mur Dueñas offers the results of contrasting morphological and syntactic elements in narrative acts in 48 English and Spanish research articles, demonstrating, by means of the study of this corpus, that in Spanish impersonal constructions are preferred over personal references; moreover, adverbs do not appear as frequently as in the 24 English articles analysed.

The second section of this volume presents a set of chapters in which assumptions about language are essentially core to the professions they explore. They range from more to less represented genres, that is, from legal and scientific areas to photographic narratives and aviation catastrophes. In "Traversing Legal Narratives", Ruth Breeze exposes how both the principle of stare decisis and the inductive method imply a "central discursive act" (p. 343); in fact, 'case stories' for current law practice and 'inductively-derived principles' for potential new law generation cannot pass unnoticed. For the purpose of her careful analysis, Breeze uses 'narrative' and 'story-telling' as synonymous, interestingly providing the figure of the judge with the role of "master narrator with a hermeneutic role" (p. 361). Yiannis Gabriel considers the technique of storytelling from a different perspective. "Researchers as Storytellers: Storytelling in Organizational Research" places the stress on academic discourses produced by researchers. This article is different from the rest as storytelling is analysed through storytelling itself, offering the reader a metastorytelling experience.

Regarding the legal field, both Patrizia Anesa's "Multiple Narratives in Arbitration Processes" and Ismael Arinas Pellón's "How do you Read a U.S. Patent? Motivation for Descriptions of Intellectual Property and its 'Metes and 
Bounds'" pinpoint interesting aspects in their respective corpora. Anesa has dealt with an area characterised by its confidentiality. Her sample might be small, but it constitutes a significant example of narratives in arbitration proceedings. Arinas, following Bazerman (1999), describes a process under an institutional framework and defines patents as "part of a process institutionalised to obtain property" (p. 476). Other professional genres covered in this section include "Narratives in Reports about Climate Change", in which Kjersti Fløttum introduces their "many varieties and genres, through different channels and voices" (p. 279) and asks for more research on this field. "The Tell and Show of Aviation-Catastrophe Synopses", by one of the editors of this collection, Carmen Sancho Guinda, explores narrative authorship, storytelling and the derived concept of tellability through the National Transportation Safety Board of the United States (NTSB). In the medical field, two articles converge on the importance of narrative. F. Salager-Meyer, M. Á. Alcaraz Ariza and M. Luzardo Briceño expose in "The Medical Narrative from a Diachronic Perspective (1840-2009): Titling Practices and Authorship" how case report narrative titles have evolved over the years. This study considers 180 randomly selected titles from 1840 to 2009, in which twenty-nine variables are identified and classified into a numerical/quantitative group and a categorical/qualitative group. The second article dealing with medical practice nowadays is Marco de Martino's "Illness Narratives: Gender and Identity in Patients' Accounts", which assesses gender and illness using a corpus based on oral illness narratives of UK patients; the first sub-corpora covers cancers affecting primary and secondary sexual organs while the second contains a miscellany of conditions. Quantitative and qualitative analyses present different conceptualisations in female and male discourses taking into account pragmatic devices and reflecting different narratives according to gender and age.

The last two chapters focus on the representation of image and text, which are recurrent in several types of professional documents. Elizabeth De Groot examines photography and its narrative through the role of complementary images and text in "Getting the Picture in Annual Reports: A Reflection on the Genrebased Analysis of Photographic Narrative". Multimodal narrations are at the basis of her analysis in which companies' annual reports constitute the medium for this type of narratives that have a core function. Isabel Corona Marzol explores two hundred printed obituaries from the four leading British newspapers in "Lives in Retrospective: the Journalistic Obituary". The obituary as a genre has been mainly adopted and exploited by writers (as The Society of Professional Obituary Writers illustrates), though it is almost absent from studies, with the exception of Mushira Eid's study, The World of Obituaries: 
Gender Across Cultures and Over Time (2002). This is certainly an interesting chapter, since the English-speaking world, unlike the Spanish, has cultivated obituary writing as part of journalistic practice.

It is a commonplace to say that words derive their meanings from the contexts in which they are uttered or written down. Narratives in Academic and Professional Genres demonstrates that the interrelations between two areas can illuminate different aspects which otherwise would have only been conceived independently. Narratology, Discourse and Genre Analysis represent valuable theoretical approaches to academic, professional and personal accounts. The areas and strategies covered in this volume offer a multiplicity of voices, analyses, experiences, and conclusions which nurture each other, providing the reader interested in ESP/EAP, Applied Linguistics or Corpus Studies with a diverse compilation. Data extracted from specific corpora can be limiting in scope and perhaps too generalising in their conclusions at times, however, they constitute a necessary task for the opening up of new research papers. Even though, as some critics are quick to point out, one of the problems with this diverse compilation is that analyses drawn from specific samples tend to restrict their range of application, the result fulfils their aims advertised on the blurb: "fresh research windows are opened on the study of academic and professional interactions".

\section{About the authors}

Dr. Yolanda Morató currently teaches in the Department of Modern Philology at the University of León. She works on Modernist Literature and the Arts, also contributing to international projects in the fields of translation, teaching methodologies, and employability or soft skills. Before earning her European PhD in English ( $U$ of Seville), she received two BAs in English and Hispanic Studies ( $U$ of Huelva and $U$ of Seville, respectively) and three MAs: Modern Literatures in English (Birkbeck College, $U$ of London); University Teaching ( $U$ of Huelva); and Translation and Interculturality ( $U$ of Seville). She took postgraduate courses and taught at Harvard University (2002-2004), where she was awarded the Harvard Certificate of Distinction in Teaching. Her articles, reviews and translations have been published in national and international journals such as EJES, Revue Française de Civilisation Britannique, The English Messenger, Atlantis, Oceanide, ES, and Quaderns de filologia. 


\section{Book Review Article history}

Paper received: $19^{\text {th }}$ February 2016

Paper received in revised form and accepted for publication: $30^{\text {th }}$ April 2016

\section{References}

Biber, Douglas and Conrad, Susan (2009). Register, Genre and Style. New York, NY: Cambridge University Press.

Eid, Mushira (2002). The World of Obituaries: Gender Across Cultures and Over Time. Detroit, MI: Wayne State University Press.

Orlikowski, W. J. and J. Yates (1994). Genre repertoire: The structuring of communicative practices in organizations. Administrative Science Quarterly, 39, 541-574.

Swales, John (1990). Genre Analysis: English in Academic and Research Settings. Cambridge: CUP. 